\title{
Use of dipsticks for routine analysis of urine from children with acute abdominal pain
}

\author{
M N Woodward, D M Griffiths
}

Wessex Regional Centre for Paediatric Surgery, Southampton General Hospital, Southampton SO9 4XY

M N Woodward, house officer D M Griffiths, consultant paediatric and neonatal surgeon

Correspondence to: Mr Griffiths.

BMF 1993;306: 1512
Urinary tract infections are common in infancy and childhood and may indicate an underlying urological problem requiring treatment. The standard method for the diagnosis of urinary tract infection remains the microscopic examination and quantitative culture of a clean catch specimen of urine, but recently a dipstick has become available that tests for two markers of infection-leucocyte esterase and nitrites. Leucocyte esterase is an enzyme from neutrophils not normally found in urine and is a marker of pyuria. Nitrites are produced in urine by the bacterial breakdown of dietary nitrates. Almost all urinary pathogens reduce nitrates to nitrites except for certain pseudomonads and group B streptococci.

The dipsticks have been tested extensively in adults ${ }^{2}$ but there are few reports of their use in children. Wiggelinkhuizen et al compared dipstick testing with formal urine analysis of 1137 children and concluded that the dipsticks were a reliable screening technique. ${ }^{3}$ Other groups have also recommended that dipsticks be used instead of urine analysis for rapid screening of children for urinary tract infection since negative results for leucocyte esterase and nitrites indicated the absence of infection. ${ }^{+5}$ The routine use of dipsticks for screening children's urine is not widespread in the United Kingdom, and so we have retested them to determine whether they should be adopted for primary screening.

\section{Patients, methods, and results}

Between November 1992 and January 1993 we tested 133 urine samples with the dipsticks (Multistix 10SG, Bayer, Newbury) in our paediatric surgical department. We did not alter our pre-existing criteria for the selection of patients for urine testing: most were children with acute abdominal pain, whose urine was

Comparison of using dipstick to test urine samples for markers (leucocyte esterase and nitrites) with urine analysis. Values are numbers

\begin{tabular}{lccc}
\hline & \multicolumn{3}{c}{ Urine analysis } \\
\cline { 2 - 4 } Screening with dipstick & Infected & Not infected & Total \\
\hline Both markers positive & 10 & 0 & 10 \\
One marker positive & 2 & 14 & 16 \\
Both markers negative & 0 & 107 & 107 \\
\hline Total & 12 & 121 & 133 \\
\hline
\end{tabular}

\section{Invasive procedures in children receiving intensive care}

\author{
David P Southall, Bernadette C Cronin, \\ Hans Hartmann, Cathy Harrison-Sewell, \\ Martin P Samuels
}

Infants and children receiving intensive care are inevitably subjected to invasive procedures. The youngest of these children-the largest group receiving intensive care-may be less able than adults to limit the number of procedures performed or ensure adequate analgesia. With unresolving illness, parents may be unable to routinely tested. Their mean age was $8 \cdot 1$ years (range 1 month to 15 years), and $71(53 \%)$ were girls. About $86 \%$ of the urine samples were taken mid-stream with the rest from a bag or catheter. A positive result was recorded if more than a trace of leucocyte esterase was detected or if any colour change for nitrites occurred.

We detected 12 urinary tract infections based on criteria of $>20$ white blood cells per $\mu l$ and $>10^{8}$ organisms per litre of a pure culture. Ten of these were positive for both leucocyte esterase and nitrites, and two were positive for just leucocyte esterase. Eight samples gave inconclusive bacteriological results, and none of these registered positive for leucocyte esterase or nitrites. The table shows that the positive predictive value of the dipstick for an infection of the urinary tract was $100 \%$ if both leucocyte esterase and nitrites were detected. The dipstick's predictive value for infection was $12.5 \%(2 / 16)$ if only one of the markers was positive. The dipstick's negative predictive value was also $100 \%$ - absence of leucocyte esterase and nitrites excluded infection of the urinary tract.

\section{Comment}

Our study has confirmed previous reports that testing of urine with the dipstick is an effective method of screening urine samples for infection. The absence of both leucocyte esterase and nitrites in a urine sample confirms its sterility; the presence of one of the markers indicates a possible infection of the urinary tract; and the presence of both confirms infected urine. We advocate the use of dipsticks, particularly for children with acute abdominal pain, as a quick and reliable means of excluding infection of the urinary tract as a cause of the symptoms. A urine sample should be sent for formal urine analysis only if one or both markers are positive. We would not, however, advise the use of dipsticks as the sole means of diagnosing infection in children with more complex renal problems, when infection with non-nitrite producing organisms, such as pseudomonads and group B streptococci, is a real possibility.

A final factor for consideration is cost. Routine processing of a midstream urine specimen costs over $£ 5$, a dipstick about $15 \mathrm{p}$. If routine use of dipsticks for screening urine became widespread the financial savings would be considerable.

1 Stevens M. Screening urines for bacteriuria. Med Lab Sci 1989;46:194-206.

2 Hurlbut TA, Littenberg B. The diagnostic accuracy of rapid dipstick tests to predict urinary tract infection. Am f Clin Pathol 1991; 96:582-8.

3 Wiggelinkhuizen J, Maytham D, Hanslo D. Dipstick screening for urinary tract infection. S Afr Med $\mathcal{f}$ 1988; 74:224-8.

4 Shaw KN, Hexter MD, McGowan KI Schwartz JS Clinical evaluation of rapid screening test for urinary tract infections in children. $f$ Pediatr 1991;118:733-6.

5 Lejeune B, Baron R, Guillos B, Mayeux D. Evaluation of a screening test for detecting urinary tract infection in newborn and infants. I Clin Pathol 1991;44:1029-30

(Accepted 19 April 1993)

make objective decisions about continuing intensive care and carers may focus on system failure and lose sight of the child as a whole person. It is our impression that children having prolonged intensive care are more likely to die, survive with serious neurological problems, or behave as if they have suffered nonaccidental injury.

This pilot study attempted to audit invasive procedures and their management in a sample of children receiving intensive care.

\section{Patients, methods, and results}

During six months nurses were asked to record on special forms every invasive procedure performed on the 96 patients who spent three days or more in the 
Academic Department of

Paediatrics, North

Staffordshire Hospital,

Stoke-on-Trent

Staffordshire ST4 6QG

David P Southall, professor of

paediatrics

Bernadette C Cronin,

research nurse

Hans Hartmann, research

fellow

Cathy Harrison-Sewell,

research nurse

Martin P Samuels, consultant

paediatrician paediatric intensive care unit at the Royal Brompton Hospital. The procedures included skin punctures, insertion or removal of catheters, tubes and drains, intubation, minor surgery, and major dressings. Information on the nature and duration of the procedure, analgesia and sedation, and the response of the patient was collected.

Data were recorded on only 55 patients ( 37 boys; median age $1 \cdot 1$ years, range $0 \cdot 1-12 \cdot 5)$. Thirty four had undergone cardiac surgery, 14 had respiratory illness, and seven had a combination of these. The clinical notes were also examined for procedures that had not been recorded. No data were obtained on the remaining 41 patients.

A total of 181 invasive procedures were recorded (table). Fifty (28\%) had been done without additional analgesia or sedation, and during 36 of these the patients had grimaced or cried. An adverse response occurred in 89 procedures ( $49 \%$ of 181 ). The median duration of procedures (recorded in 172 cases) was 5 minutes (range 0.5 to 120 ). A relative was present during 36 procedures.

The clinical notes of the 55 patients disclosed an additional 318 unrecorded procedures (range one to 159 per patient), making a total of 499 . Thirty three patients with a duration of stay of three to seven days had a median of 2.0 procedures (range 1-11), and 22 with a duration of stay of eight to 265 days had median of 3.5 procedures (range $1-159$ ). Two patients had 43 and 159 procedures respectively during stays of 31 and 265 days. When last seen, the first of these was well but awaiting further surgery; the second had severe learning difficulties and cerebral diplegia at 2 years of age.

In a random sample of nine of the 41 patients admitted, but in whom no invasive procedures were charted, examination of the hospital notes identified a total of 43 procedures.

\section{Comment}

This study confirms our concerns that (a) some children receiving intensive care have a protracted

Response of patient according to type of analgesia or sedation

\begin{tabular}{|c|c|c|c|c|c|c|c|}
\hline \multirow[b]{2}{*}{ Type of analgesia } & \multicolumn{6}{|c|}{ Response to invasive procedures } & \multirow[b]{2}{*}{ Total } \\
\hline & $\begin{array}{c}\text { None } \\
\text { apparent }\end{array}$ & Grimace & Cry & $\begin{array}{l}\text { Cardio- } \\
\text { respiratory } \\
\text { arrest }\end{array}$ & Cyanosis & Unrecorded & \\
\hline Nil & 13 & 11 & 25 & & & 1 & 50 \\
\hline Analgesia or sedation & 38 & 23 & 10 & 1 & & 3 & 75 \\
\hline Local anaesthetic & 7 & 6 & 1 & & & & 14 \\
\hline Combination ${ }^{\star}$ & 9 & 1 & 1 & 1 & & 1 & 13 \\
\hline General anaesthetic & 11 & 1 & 1 & & 2 & $i$ & 16 \\
\hline Unrecorded & 6 & 3 & 2 & & & 2 & 13 \\
\hline All procedures & 84 & 45 & 40 & 2 & 2 & 8 & 181 \\
\hline
\end{tabular}

*Combination of analgesia, sedation, local anaesthetic, and/or paralysis. illness and large numbers of invasive procedures and (b) many procedures that are not preceded by additional analgesia or sedation are accompanied by crying or grimacing. Despite considerable efforts to achieve compliance retrospective review of admission records and selected hospital notes showed that a large proportion of patients (41 of 96) and procedures were not audited. This was not unexpected, as the unit was often extremely busy and understaffed. Our subsequent analysis of the hospital notes must also have greatly underestimated the number of intravenous and intraarterial lines placed, with no hope of identifying the number of skin punctures required.

Other studies have led to progress in the use of analgesia and sedation during neonatal intensive care. ${ }^{1-3}$ There is a need for similar research in older children, ${ }^{4}$ particularly with respect to the long term psychological consequences of prolonged intensive care.

Our findings support the need for the routine and continual recording of invasive procedures. One difficulty with intensive care is the necessity for large numbers of different carers who individually spend comparatively short periods with each patient. We suggest that every child in intensive care should have a single paediatrician who cares for him or her as a whole person and who ensures that each invasive procedure is performed only when it is essential. For example, non-invasive monitoring of oxygen saturation and transcutaneous carbon dioxide pressure should be used to reduce the frequency of blood sampling, which, if essential and frequent, should be from either an indwelling arterial or central venous line inserted with adequate analgesia or sedation or entail the least discomforting technique-for example, an Autolet for capillary samples. ${ }^{5}$ With increasing numbers of procedures the risks associated with increasing drug doses may have to be accepted to ensure adequate sedation or analgesia.

Invasive procedures are inevitable in intensive care. When care becomes prolonged a child may be overwhelmed by repetitive, discomforting procedures. Avoiding them whenever possible from the outset along with their audit by the child's own paediatrician should be essential components of paediatric intensive care. Further research is required into the efficacy of analgesia and sedation as well as the long term psychological effects of prolonged intensive care.

1 Anand KJS, Hickey PR. Pain and its effects in the human neonate and fetus. N Engl F Med 1987;317:1321-9.

2 Anand KJS, Hickey PR. Halothane-morphine compared with high dose sufentanil for anaesthesia and postoperative analgesia in neonatal cardiac sufentanil for anaesthesia and postop

3 Tohill J, McMorrow O. Pain relief in neonatal intensive care. Lancet 1990;336: 569 .

4 McGrath PJ, Unruh AM. Pain in children and adolescents. New York: Elsevier, 1987.

5 Harpin VA, Rutter N. Making heel pricks less painful. Arch Dis Child 1983;58:226-8.

(Accepted 8 April 1993)

\section{ANY QUESTIONS}

Is there any evidence that $\beta$ blockers help people to give up cigarette smoking?

There do not seem to be any published studies that have examined whether $\beta$ blockers help people who are trying to stop smoking. The rationale for any possible effect is not clear. While symptoms such as anxiety and restlessness form part of the nicotine withdrawal syndrome, their manifestation is mainly in terms of self report of affective state rather than more somatic signs such as tremor or palpitations. There are reports that $\beta$ blockers may reduce the risk of death after acute myocardial infarction in cigarette smokers but no indication that this benefit might be achieved through facilitating cessation.'-MARTIN JARVIS, Imperial Cancer Research Fund, London

1 Jafri SM, Tilley BC, Peters R, Schultz LR, Goldstein S. Effects of cigarette smoking and propranolol in survivors of acute myocardial infarction. $\mathrm{Am}$ f Cardiol 1990;65:271-6. 